\title{
An Updated Systematic Review of Neuroimaging Studies of Children and Adolescents with Perinatally Acquired HIV
}

\author{
Kayla A. Musielak ${ }^{1}$. Jodene Goldenring Fine ${ }^{1}$
}

Received: 9 June 2015 / Revised: 1 October 2015 / Accepted: 7 October 2015 / Published online: 4 November 2015

(C) American Academy of Pediatric Neuropsychology 2015

\begin{abstract}
A recent review of neuroimaging studies related to perinatally acquired human immunodeficiency virus (HIV) infection by Hoare and colleagues (Metabolic Brain Disease 29:221-229, 2014) included studies published between 1995 and 2012, with all but two studies being published in or before 2006. Although the review synthesized the extant research available at the time, the findings may not be relevant to the current population of children and adolescents who are HIV positive due to more recent advances in antiretroviral medication and new medication guidelines. As such, the purpose of this paper was to extend the findings of Hoare and colleagues by reviewing the imaging literature that has been published since 2012 and to compare the more recent studies to the earlier ones. The results were that 11 articles met criteria for inclusion in this review. The majority of studies were crosssectional and analyzed MRI data.. Samples included children and adolescents of nearly all ages. Studies differed widely on the inclusion and reporting of clinical variables. Imaging was focused primarily on white matter, and white matter issues such as lesions, poor tract integrity, and reduced volume were found in samples of children and adolescents of various age ranges. Two studies compared the cognitive functioning of children with HIV to controls, and both found that children with HIV perform more poorly than their non-infected peers on various cognitive tasks. Based on the findings of this review, recommendations for future imaging research are made.
\end{abstract}

Keywords HIV · Imaging $\cdot$ Children $\cdot$ Review

Kayla A. Musielak

musielak@msu.edu

1 Michigan State University, 620 Farm Lane, Room 435, East Lansing, MI 48824, USA

\section{Introduction}

Much progress has been made in recent decades regarding the prevention of transmission of human immunodeficiency virus (HIV) infection. Despite this, approximately 3.2 million children and adolescents around the world are living with perinatally acquired HIV (World Health Organization 2015b). With treatment, these children will be more likely to survive into adulthood (Patel et al. 2008). Modern standard treatment typically consists of a combination of three medicines referred to as highly active antiretroviral therapy (HAART; World Health Organization 2015a). Research on the potential effects of HAART to mitigate problems with neurocognitive development and performance is mixed in general and is particularly limited in children. However, current research suggests that neurocognitive difficulties persist in the era of HAART medications (Liner et al. 2010; Whitehead et al. 2013). It is, therefore, important for researchers and clinicians to understand the nature of the neurocognitive difficulties children with HIV may experience.

According to Ellis et al. (2009), HIV damages the central nervous system in two ways. The virus infects certain cells of the central nervous system and produces various proteins that can damage neuronal cells and interfere with their functioning. In turn, the immune response to the virus by the cells may be impaired and inflammation can then persist (Wood et al. 2009). Consequently, there may be neurological sequelae of HIV that result in the neurocognitive symptoms observed in children with HIV. Another issue to consider is the potential role of HAART medications in the neurocognitive development of children, as research has also found that antiretroviral medications may be toxic to brain cells (Robertson et al. 2012). As discussed by Liner et al. (2010), antiretrovirals can lead to mitochondrial dysfunction, destabilization of neuronal intracellular homeostasis, reduction of cell membrane 
potential, and dendritic beading and pruning. Thus, it may be that not only the disease but also the treatment may contribute to the progressive neurocognitive challenges seen in children with HIV.

Understanding the neurological implications of HIV and related variables is critical to the treatment of these vulnerable children. The brain-behavior relationships and the etiology of the difficulties observed in the homes, schools, and playgrounds of children with HIV are not currently well defined, particularly regarding the relative roles of the disease and the treatment of the disease. Neuroimaging may also be useful in this respect. Comparing neuroimaging findings in adult samples to those in child samples may help scholars and clinicians to better understand neurological effects over time and development. Finally, Hoare et al. (2014) argue that clinical criteria on the spectrum of HIVassociated neurocognitive disorders (HANDs) that are presently available for adults with HIV are also necessary for children and that neuroimaging research will need to play a role in the development of these criteria.

There is a relatively small body of literature regarding the neurological implications of HIV in children, including a recent review of the literature by Hoare et al. (2014) who systematically examined the results of neuroimaging studies of HIV in children. The review search resulted in a total of 11 articles that met the authors' criteria for inclusion. Their overall findings were that the most frequent neurological abnormalities included ventricular enlargement, atrophy of cortical and subcortical regions, damage to the basal ganglia and corpus callosum, and abnormalities in both white and gray matter in the frontal lobe. Although this review summarized the extant literature available at the time and highlighted several specific neurological implications of HIV, there is an important limitation to the review. All but two of the studies included in Hoare's review were published in or before 2006, the earliest of which was published in 1995. Of the two studies published after 2006, one was published in 2011 and one in 2012. It is unclear whether the findings of Hoare et al. (2014) are relevant to the current population of HIVpositive children and adolescents because of advances in antiretroviral medication and new medication guidelines.

Guidelines for treating childhood HIV have changed substantially over the past several years. Adjustments of the criteria for initiation of treatment and the changing of regimens have occurred. For example, in 2006, the World Health Organization (WHO)'s recommendations were to treat all infants and children with HIV who were in the WHO-specified pediatric stages 3 and 4 (see Table 1 for WHO staging classification). Health professionals were advised to use $\mathrm{T}$ cell-bearing cluster of differentiation 4
(CD4) receptor or total lymphocyte count (TLC) data to inform treatment decisions for those in stages 1 and 2, but to not treat those without CD4 data in stage 1 (World Health Organization 2006). However, in 2008 and more recent guidelines, the WHO has recommended that all children with HIV under 1 year of age be provided with antiretroviral treatment (ART). The recommendations for children older than 1 year have also changed across the recent iterations of the guidelines (World Health Organization 2008, 2010, 2013). In general, the recommendations for when to initiate ART have become more inclusive, such that all children under the age of 5 should receive ART regardless of clinical stage or CD4 data under the 2013 guidelines (World Health Organization 2013). The presence of ART during infancy and early childhood would likely influence the impact of the virus on the child's developing central nervous system and also influence the central nervous system by way of effects from immune responses and medication. This would suggest that newer treatment regimens and administration protocols may result in different neurological sequelae in the brains of children with HIV.

As mentioned previously, only two studies examined in the review of Hoare et al. (2014) were published after 2006. This suggests that the majority of studies reviewed included samples who potentially have had very different experiences with ART than the population of children who are currently living with HIV. As such, the purposes of the present study are (1) to extend the findings of Hoare and colleagues' review to include more recent studies and (2) to compare the findings of the recent studies to those of the earlier studies.

\section{Methods}

The studies for this review were found by searching for articles published between 2012 and April 2015. A search was conducted using combinations of the following terms: human immunodeficiency virus (HIV), HIV, acquired immunodeficiency virus (AIDS), AIDS, pediatric, children, adolescents, neuroimaging, brain imaging, magnetic resonance imaging (MRI), MRI, functional magnetic resonance imaging (fMRI), fMRI, diffusion tensor imaging (DTI), and DTI. Article sources included PsycINFO, PsycARTICLES, and PubMed. Studies were included in this review if they were in English, described original research, utilized neuroimaging techniques, and included a sample of at least five participants in which the majority of participants were less than 18 years old. Children had to have acquired HIV during the perinatal period. The authors of the present study believe that it is important to include the studies with samples involving a minority 
Table 1 Clinical stages of HIV infection for children (World Health Organization 2007)

\section{WHO clinical stage}

\begin{tabular}{|c|c|}
\hline 1 & $\begin{array}{l}\text {-Asymptomatic } \\
\text {-Persistent generalized lymphadenopathy }\end{array}$ \\
\hline 2 & $\begin{array}{l}\text {-Unexplained persistent hepatomegaly and splenomegaly } \\
\text {-Papular itchy skin eruptions } \\
\text {-Extensive skin warts } \\
\text {-Extensive molluscum contagiosum } \\
\text {-Recurrent oral ulcerations } \\
\text {-Unexplained persistent parotid gland enlargement } \\
\text {-Linear gingival erythema } \\
\text {-Herpes zoster } \\
\text {-Recurrent or chronic respiratory tract infections (sinusitis, otorrhea, tonsillitis, otitis media) } \\
\text {-Fungal nail infections }\end{array}$ \\
\hline 3 & $\begin{array}{l}\text {-Moderate unexplained malnutrition not responding to standard therapy } \\
\text {-Unexplained persistent diarrhea for longer than } 14 \text { days } \\
\text {-Unexplained persistent fever above } 37.5^{\circ} \mathrm{C} \text { (intermittent or constant for more than } 1 \text { month) } \\
\text {-Persistent oral candidiasis (outside the first } 6-8 \text { weeks of life) } \\
\text {-Oral hairy leukoplakia } \\
\text {-Acute necrotizing ulcerative gingivitis or periodontitis } \\
\text {-TB lymphadenopathy } \\
\text {-Pulmonary tuberculosis } \\
\text {-Severe recurrent presumed bacterial pneumonia ( } 2 \text { or more episodes in a 6-month period) } \\
\text {-Symptomatic lymphoid interstitial pneumonitis (LIP) } \\
\text {-Chronic HIV-associated lung disease, including bronchiectasis } \\
\text {-Unexplained anemia }(<8 \mathrm{~g} / \mathrm{dl}) \text {, neutropenia }\left(<500 / \mathrm{mm}^{3}\right) \text {, or thrombocytopenia }\left(<50,000 / \mathrm{mm}^{3}\right)\end{array}$ \\
\hline $4^{a}$ & $\begin{array}{l}\text {-Unexplained severe wasting, stunting, or severe malnutrition not responding to treatment } \\
\text {-Pneumocystis carinii (jirovecii) pneumonia } \\
\text {-Recurrent severe presumed bacterial infections (e.g., empyema, pyomyositis, bone or joint infections, } \\
\text { meningitis, sepsis, but excluding pneumonia) } \\
\text {-Toxoplasmosis of the brain } \\
\text {-Cryptosporidiosis with diarrhea }>1 \text { month } \\
\text {-Isosporiasis with diarrhea }>1 \text { month } \\
\text {-Cryptococcosis, extrapulmonary } \\
\text {-Cytomegalovirus of an organ other than the liver, spleen, or lymph node } \\
\text {-Chronic herpes simplex infection (orolabial or cutaneous for }>1 \text { month) } \\
\text {-Progressive multifocal leukoencephalopathy } \\
\text {-Any disseminated endemic mycosis } \\
\text {-Candidiasis of the esophagus, trachea, and bronchus } \\
\text {-Atypical mycobacteriosis, disseminated or lungs } \\
\text {-Extrapulmonary tuberculosis excluding TB lymphadenopathy } \\
\text {-Lymphoma (cerebral or B cell non-Hodgkin) } \\
\text {-Kaposi's sarcoma } \\
\text {-HIV encephalopathy } \\
\text {-HIV-associated cardiomyopathy or HIV-associated nephropathy }\end{array}$ \\
\hline
\end{tabular}

${ }^{\text {a }}$ Some additional specific conditions can also be included in regional classifications (such as reactivation of American trypanosomiasis (meningoencephalitis and/or myocarditis) in the WHO Region of the Americas, disseminated penicilliosis in Asia, and HIV-associated rectovaginal fistula in Africa)

of children just above 18 years of age because this transitional period from teenage years to adulthood is clinically relevant to those who work with pediatric populations, and because brain maturation occurs well into young adulthood (Sowell et al. 2001) and there is much heterogeneity in brain development among individuals (Gogtay et al. 2004).

The first author evaluated the titles and abstracts of each article to determine whether they met criteria for inclusion in this review. Of the 108 articles that resulted from the search,
56 were not related to HIV or the individuals were not HIV positive. Four duplicate articles were then removed from the sample. The remaining 48 articles included 5 review articles, 3 articles not about humans, 16 articles with adult participants, 6 articles that did not include neuroimaging data, and 7 that included fewer than five participants. Only 11 articles met criteria for inclusion. The 11 studies included in this review were analyzed in order to better understand the type of studies conducted, the neuroimaging techniques used, the variables assessed, the samples included, and the findings obtained. 


\section{Results}

\section{Design}

Please see Table 2 for a summary of each study. The studies in this review were primarily cross-sectional in design. One article (van Arnhem et al. 2013) included a longitudinal substudy in the paper for which a portion of the original sample was randomly selected for follow-up. Two studies (Izbudak et al. 2013; Mariam and Assefa 2012) also included longitudinal data for the children in their studies when it was available. Three studies were reportedly retrospective in design (Izbudak et al. 2013; Mariam and Assefa 2012; van Arnhem et al. 2013) and utilized data collected over a period of time between 8 (Mariam and Assefa 2012) and 18 years (van Arnhem et al. 2013). Two studies (Andronikou et al. 2014, 2015) were reportedly retrospective within a current prospective study. Only two studies (Ackermann et al. 2014; Donald et al. 2015) clearly indicated that their data were from a prospective study. Four studies (Andronikou et al. 2015; Hoare et al. 2012; Nagarajan et al. 2012; Sarma et al. 2014) compared children with perinatally acquired HIV to non-infected peers.

\section{Aim}

In general, the aims of the studies were to examine neurological indications of disease. Four studies (Ackermann et al. 2014; Andronikou et al. 2014; Hoare et al. 2012; Nagarajan et al. 2012) also aimed to examine brain-behavior relations by assessing cognitive functioning by way of standardized cognitive or developmental assessment.

\section{Technique}

Several neuroimaging modalities were used in the studies reviewed. Four of the articles included in the present study (Ackermann et al. 2014; Andronikou et al. 2014, 2015; Sarma et al. 2014) utilized only MRI techniques. Four studies (Donald et al. 2015; Izbudak et al. 2013; Mariam and Assefa 2012; van Arnhem et al. 2013) used a combination of computed tomography (CT), MRI, magnetic resonance angiogram (MRA), ultrasound, and cerebral angiogram. Two studies (Hoare et al. 2012, 2015) used DTI, and one used magnetic resonance spectroscopy (MRS; Nagarajan et al. 2012).

\section{Age}

The studies included samples of children of various ages. Three studies (Ackermann et al. 2014; Andronikou et al. 2014, 2015) included young children generally around the age of 2 , but the range comprised children as young as several months old up to 4 years. Three studies (Donald et al. 2015;
Mariam and Assefa 2012; van Arnhem et al. 2013) had a median of sample ages of around 5 years but included very young children in middle to late childhood. Two studies (Hoare et al. 2012, 2015) were completed with samples with mean ages of around 9 to 10 years. In one of these studies (Hoare et al. 2015), the age range of participants was 6 to 15 years, and in another (Hoare et al. 2012), the range was only 8 to 12 years. Three studies (Izbudak et al. 2013; Nagarajan et al. 2012; Sarma et al. 2014) included samples with mean ages in the mid to late teens, but the range spanned from childhood into adulthood. Sample sizes ranged from a total of 8 participants (Izbudak et al. 2013) to 87 participants (in this study, only 49 had imaging data; Donald et al. 2015).

\section{Antiretroviral Therapy}

A primary interest of the present study was to examine the presence of and information provided about ART in the reviewed studies. In the current and following sections, ART refers generically to antiretroviral treatment or is used when authors did not specify monotherapy versus combination therapy whereas HAART refers specifically to combination antiretroviral treatment. Ten studies (Ackermann et al. 2014; Andronikou et al. 2014, 2015; Donald et al. 2015; Hoare et al. 2015; Izbudak et al. 2013; Mariam and Assefa 2012; Nagarajan et al. 2012; Sarma et al. 2014; van Arnhem et al. 2013) included participants with ART experience. Of these, only three studies (Ackermann et al. 2014; Hoare et al. 2015; van Arnhem et al. 2013) provided the ART medications most often provided to the children. Eight of the ten studies (Ackermann et al. 2014; Andronikou et al. 2014, 2015; Hoare et al. 2015; Izbudak et al. 2013; Nagarajan et al. 2012; Sarma et al. 2014; van Arnhem et al. 2013) specifically mentioned having samples in which at least some children were receiving HAART. Ackermann et al. (2014) included children who had been participating in another study in which they were assigned to differing ART arms, such that some children were not on ART and some were on ART for differing lengths of time. In the study by Donald et al. (2015), all but one child was on ART at the time of assessment. Additionally, participants of Izbudak et al. (2013) were not all on HAART at the time of initial imaging. Mariam and Assefa (2012) reported that all participants had been prescribed HAART at one time, and the majority of participants were still on HAART. One study (Hoare et al. 2012) included only children who were HAART naive. None of the studies reported data regarding ART adherence.

\section{Other Clinical Variables}

In addition to ART, the studies also varied on the inclusion of other clinical variables in statistical analyses and the reporting of descriptive sample data. The CD4 and viral load data were 


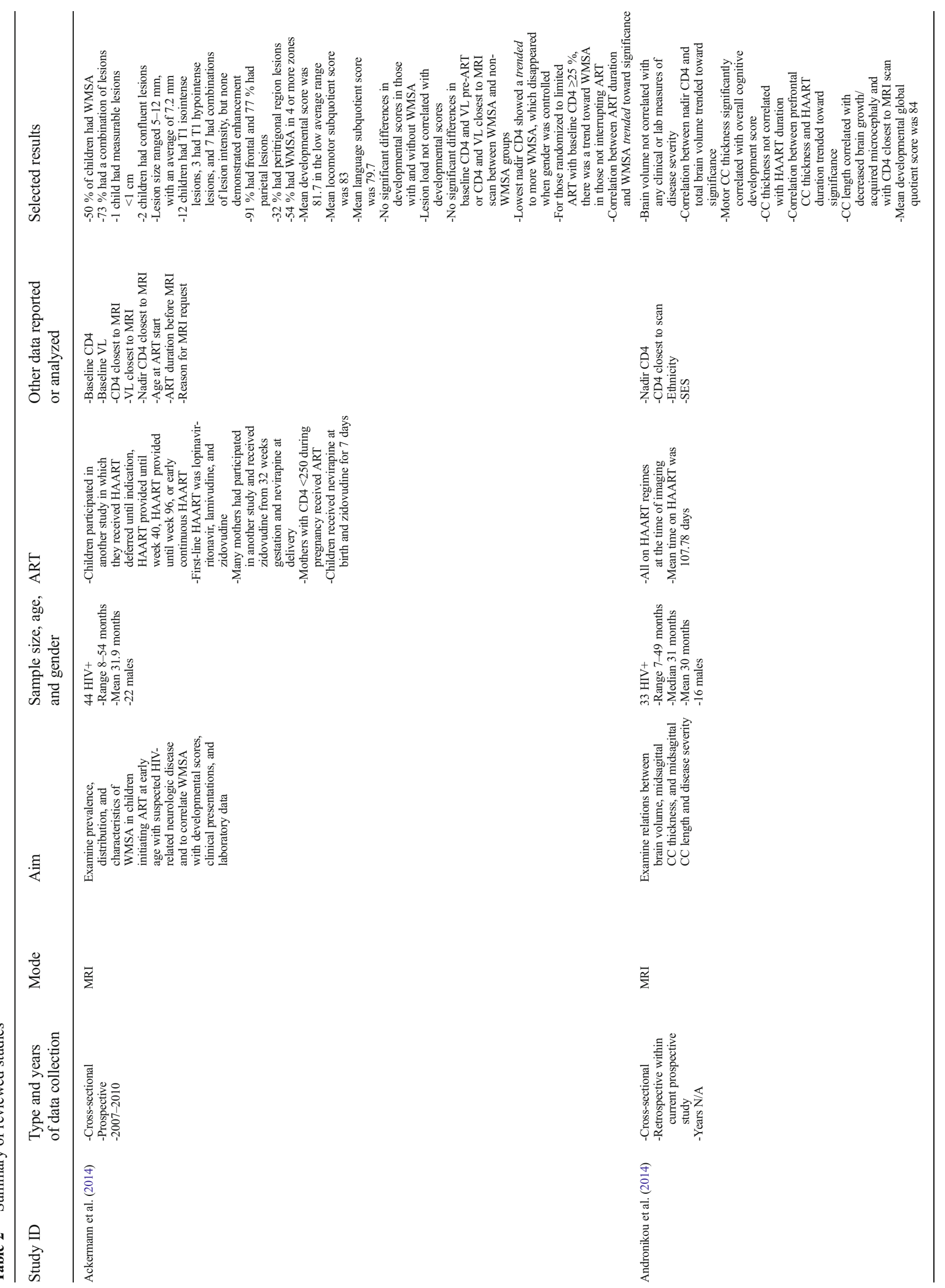




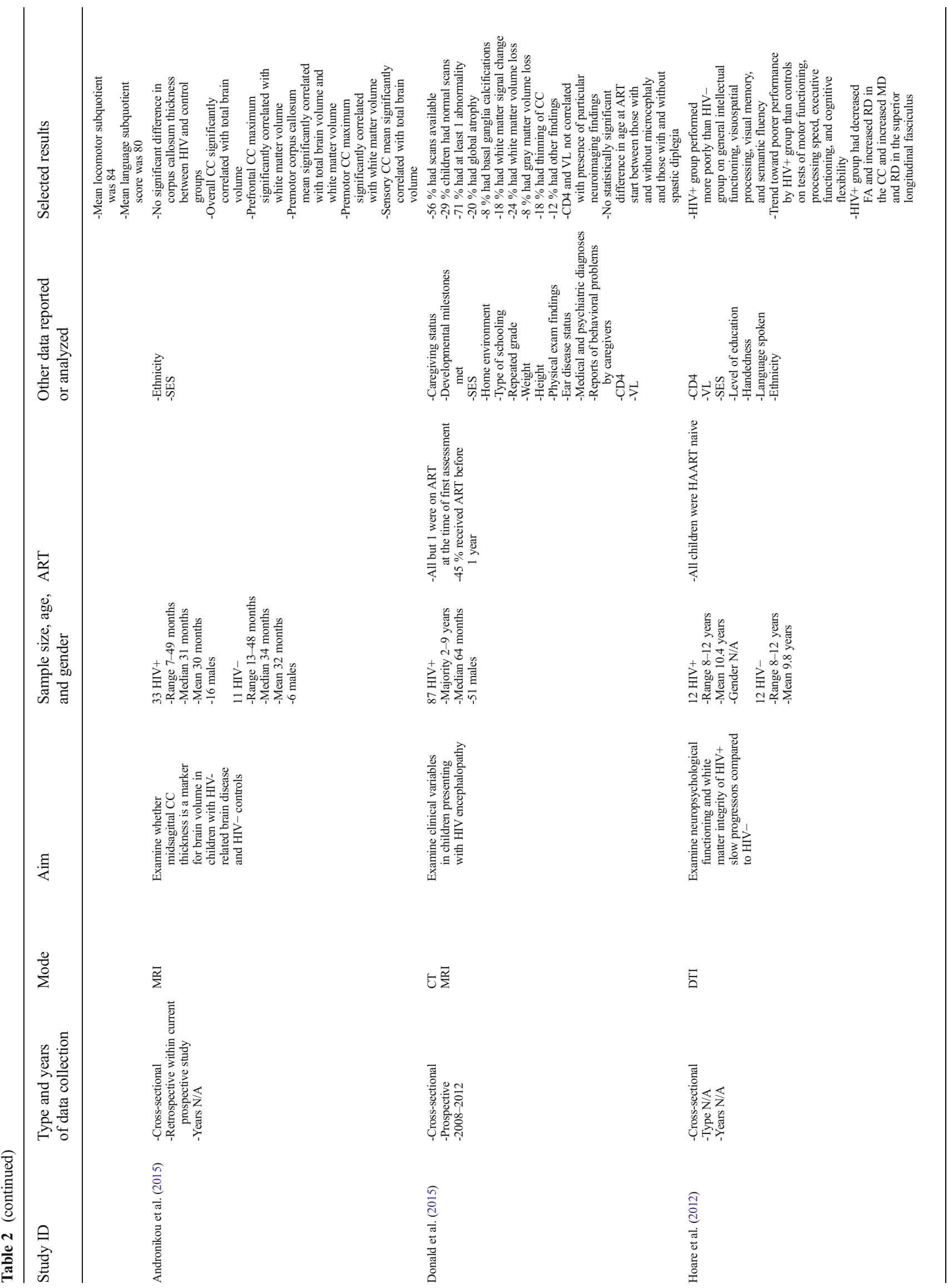




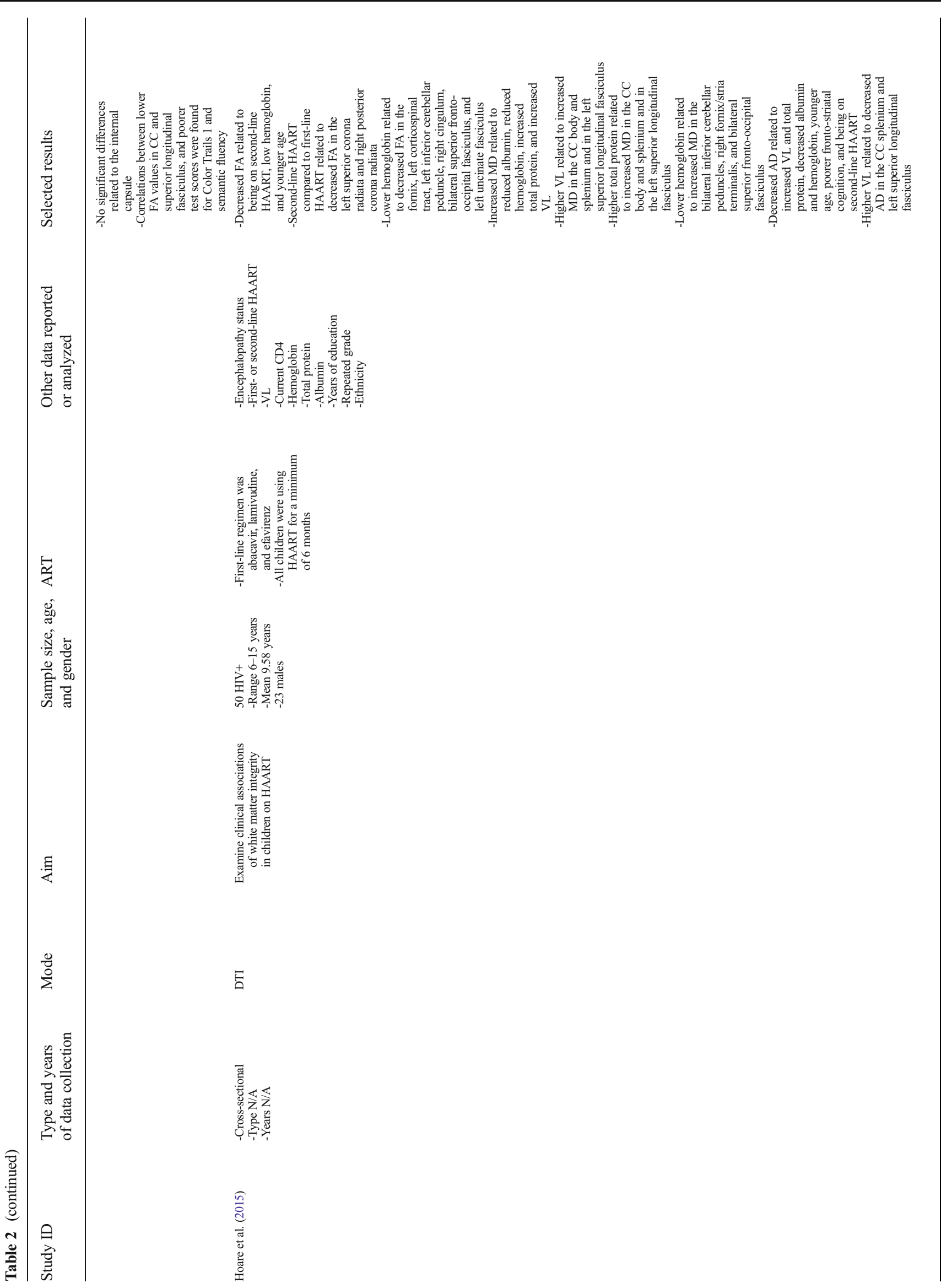




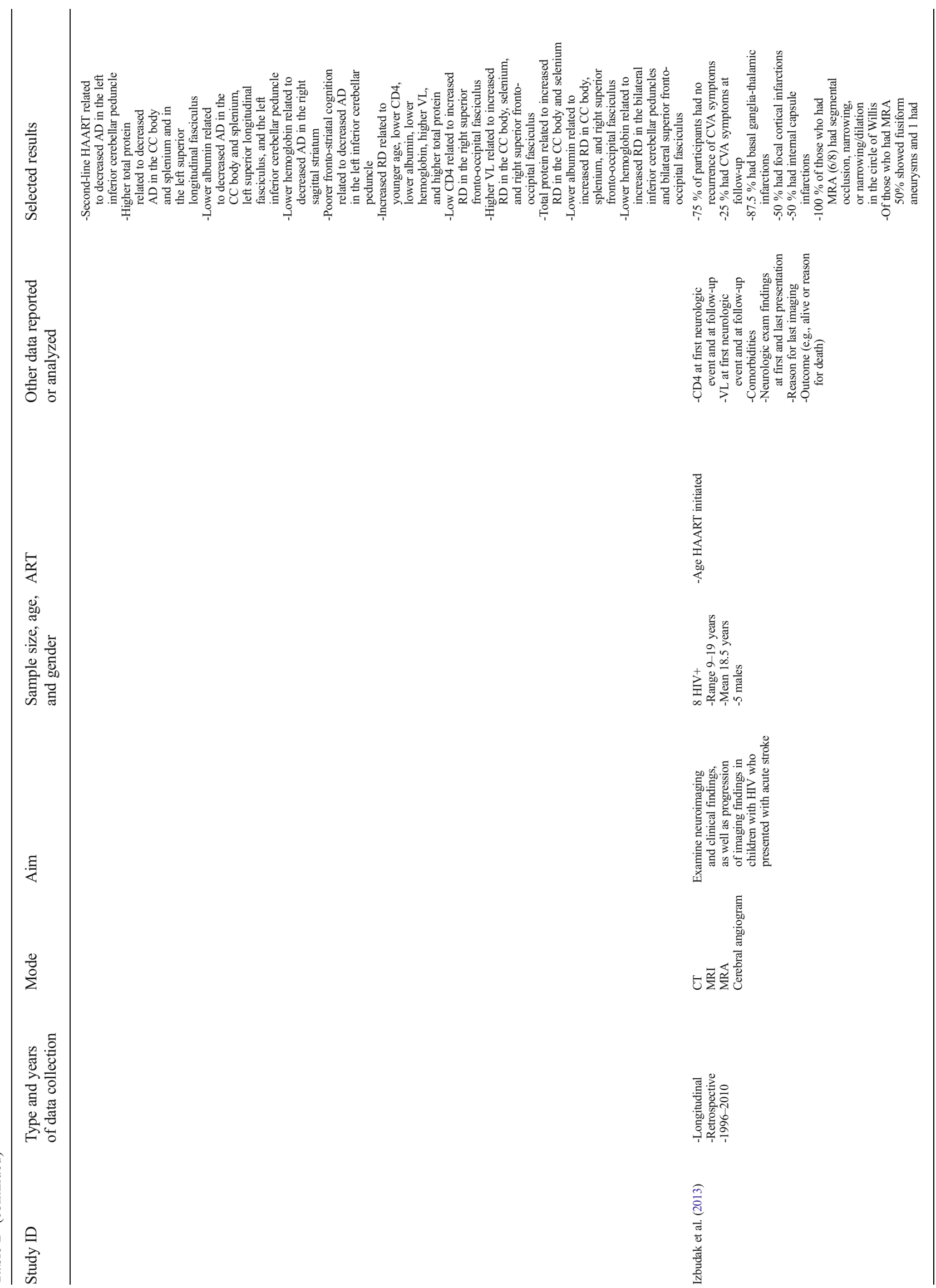




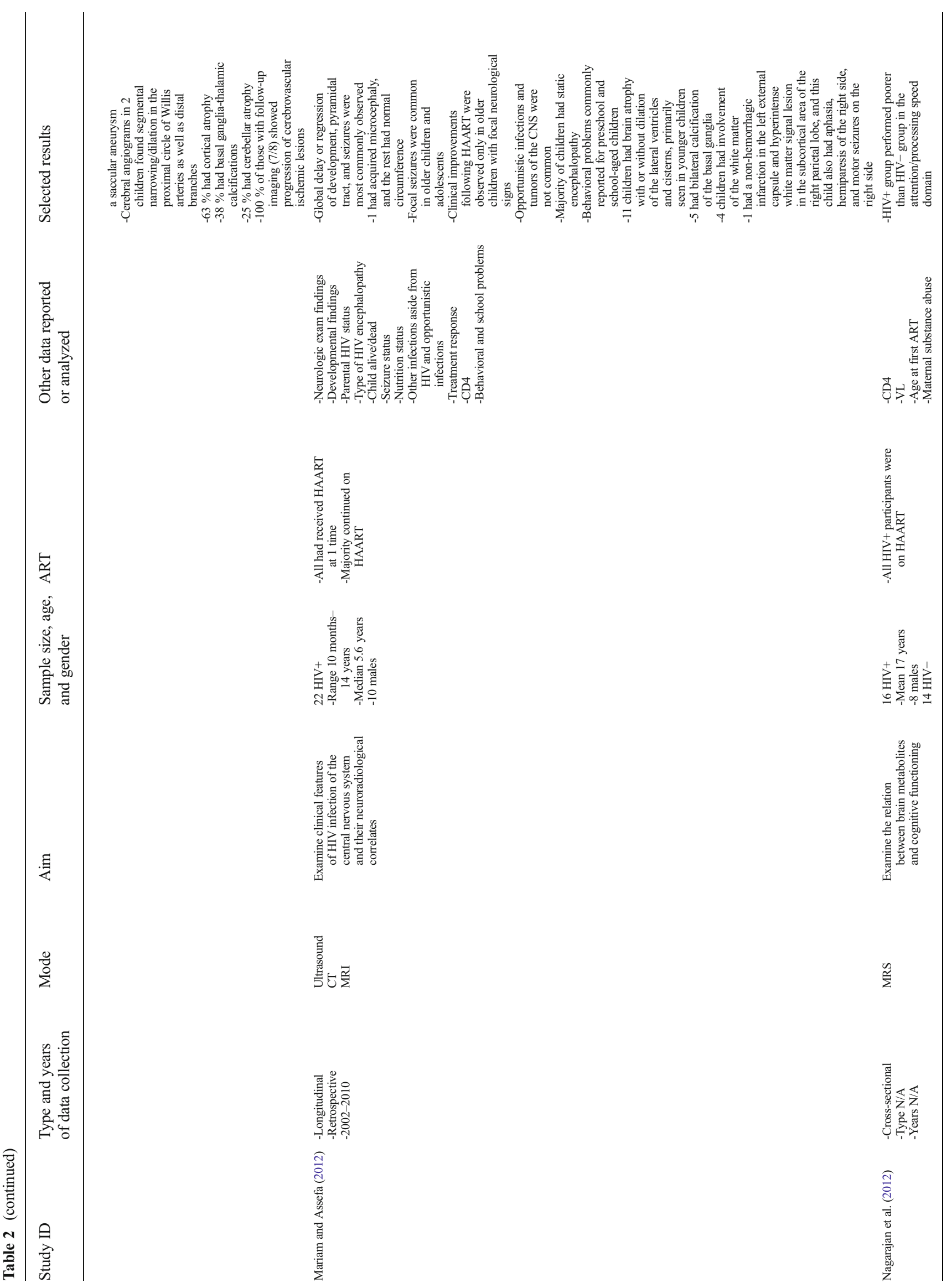




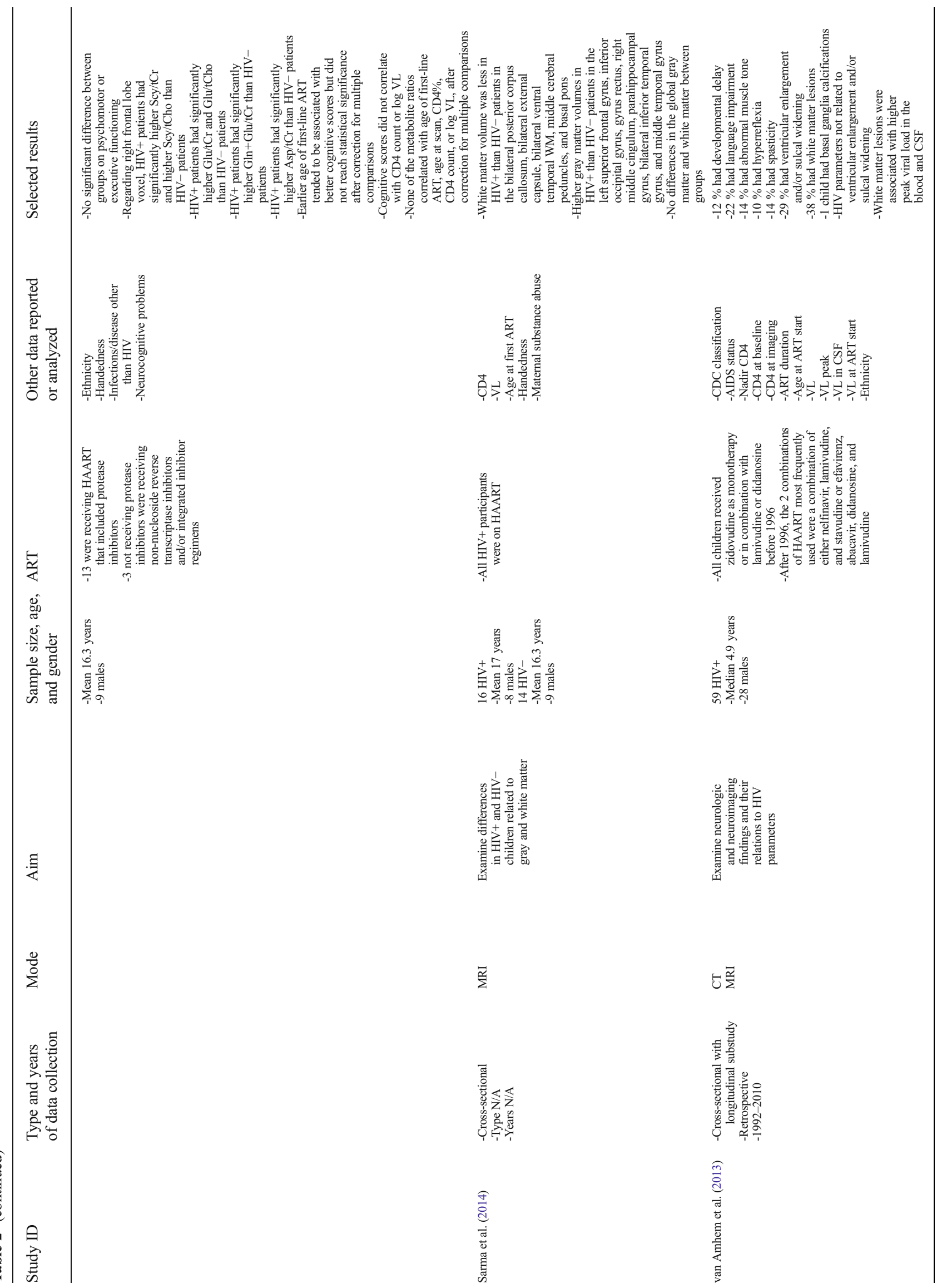




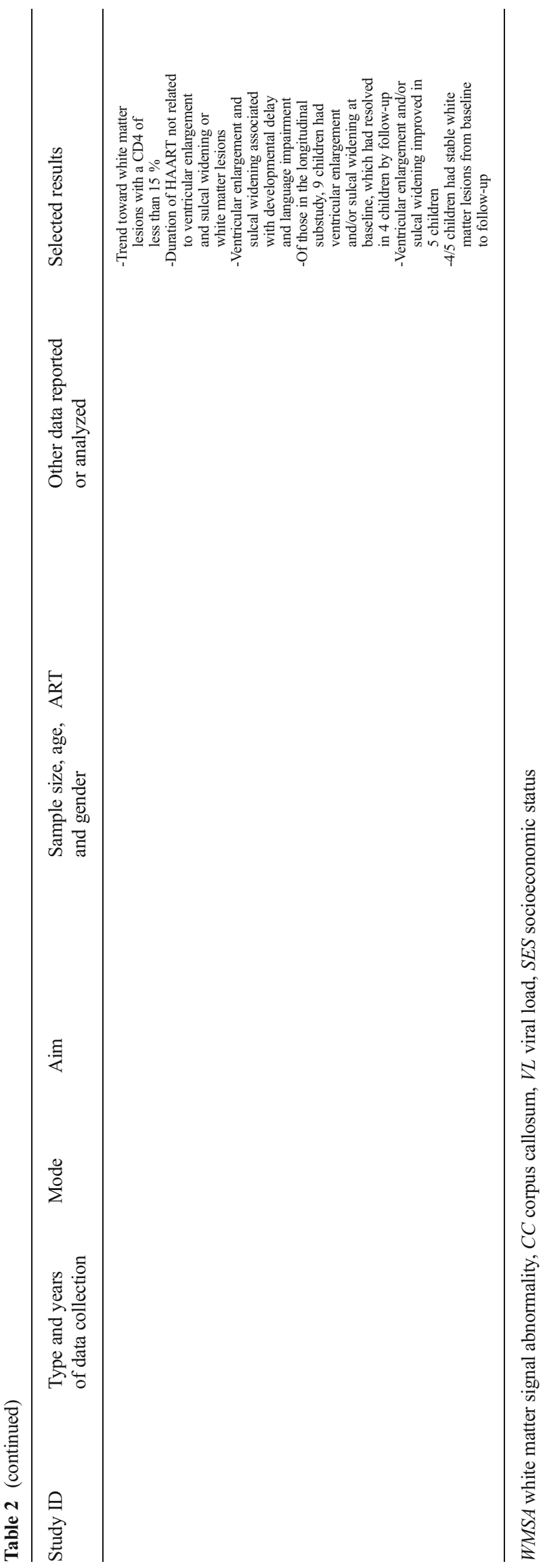

the most commonly reported clinical data and were reported by all but one study (Andronikou et al. 2015). Notably, the exact type of data regarding CD4 and viral load differed among studies. For example, some studies reported nadir CD4 and some included CD4 data at various time points (e.g., at baseline or closest to scan date). The reporting of different clinical parameters across studies makes it difficult to ascertain which parameters are most predictive of neurological damage.

There was an extensive range of other data reported. Examples of other medically related variables included in the studies reviewed are age at first ART, maternal substance use, height, weight, encephalopathy status, protein levels, and CDC classification. Examples of other clinically relevant but non-medical variables included home environments, caregiving status, and developmental milestones met. Although there was a range of other clinical data reported, few studies reported a comprehensive set of demographic, health-related, and other variables. For example, only three studies (Donald et al. 2015; Hoare et al. 2015; Mariam and Assefa 2012) reported nutrition- or growth-related data, and four reported socioeconomic information (Andronikou et al. 2014, 2015; Donald et al. 2015; Hoare et al. 2012).

\section{Imaging Results}

Not all children in the included studies had observable neuroradiological problems. In the study by Donald et al. (2015), $29 \%$ of children had normal scans. Half of the participants in the study by Ackermann et al. (2014) did not have white matter signal abnormalities. Furthermore, ventricular enlargement and/or sulcal widening was found in $29 \%$ and white matter lesions were found in $38 \%$ of participants in the study by van Arnhem et al. (2013), suggesting that there were many children who did not demonstrate these neurological problems.

White matter was examined in 9 out of 11 studies. White matter involvement ranging from white matter signal abnormalities and lesions (Ackermann et al. 2014; Donald et al. 2015; Izbudak et al. 2013; van Arnhem et al. 2013) to reduced white matter (Donald et al. 2015; Sarma et al. 2014) to decreased white matter integrity (Hoare et al. 2012) was frequently observed. Ackerman and colleagues found that $73 \%$ of children with white matter signal abnormalities/ lesions had a combination of lesions ranging from pinpoint lesions to confluent lesions. Lesions in subcortical and deep white matter were most commonly in the frontal $(91 \%)$ and parietal $(77 \%)$ areas. These children had generally received ART from an early age but were suspected to have neurological disease. White matter lesions were also observed in $18 \%$ of the children with encephalopathy who were being prescribed ART at the time of participation in the study by Donald et al. (2015). Lesions were found in $38 \%$ of children in the 
study by van Arnhem et al. (2013). These children varied in terms of their experience with CDC classification and ART. In the latter two studies, it is not clear where the lesions tended to be observed. Internal capsule infarctions were observed in half of the participants, and a periventricular white matter signal abnormality was observed in one participant in the study by Izbudak et al. (2013). All of these participants experienced an acute stroke, and they generally had high viral loads at the first assessment. Finally, a hemorrhagic infarction in the external capsule and signal abnormality in the subcortical right parietal lobe was reported in one child who had aphasia, hemiparesis, and focal motor seizures in the study by Mariam and Assefa (2012).

Less white matter volume was observed by Sarma et al. (2014) in adolescents and young adults who were prescribed HAART at the time than in those without HIV. Areas in which volume was reduced included the bilateral posterior corpus callosum, bilateral external capsule, bilateral ventral temporal white matter, middle cerebral peduncles, and basal pons. Approximately one quarter of the children in the study by Donald et al. (2015) were prescribed ART, had encephalopathy, and experienced white matter volume loss.

Hoare et al. (2012) assessed the white matter integrity of the corpus callosum, internal capsule, and superior longitudinal fasciculus of children who were HAART naive and asymptomatic via DTI. They found that children with HIV had lower fractional anisotropy (FA) and increased radial diffusivity (RD) in the corpus callosum than children without HIV. Increased RD and mean diffusivity (MD) was found in the superior longitudinal fasciculus. No significant differences were found related to the internal capsule.

The corpus callosum was specifically examined in multiple studies (Andronikou et al. 2014, 2015; Donald et al. 2015; Hoare et al. 2012, 2015; Sarma et al. 2014). No difference in corpus callosum thickness was found in a study comparing children with HIV to non-infected children (Andronikou et al. 2015), but little is known about the demographic and clinical status of this sample. In contrast, thinning of the corpus callosum was observed in $18 \%$ of participants in one study of young children who were nearly all on ART, of which approximately half had been prescribed ART while in infancy (Donald et al. 2015). As reported previously, Hoare et al. (2012) also found decreased integrity and Sarma et al. (2014) found less volume in the corpus callosum in children with HIV compared to children without HIV.

The relations among white matter issues and clinical variables were mixed in the reviewed studies. White matter involvement was found to be related to some clinical variables including CD4 levels (Andronikou et al. 2014; Hoare et al. 2015), viral load (Hoare et al. 2015; van Arnhem et al. 2013), and second-line HAART (Hoare et al. 2015). However, van Arnhem et al. (2013) found CD $4<15 \%$ to be close but not statistically significantly related to white matter lesions and
Ackermann et al. (2014) found no difference in CD4 or viral load levels between those with white matter signal abnormalities and those without, although CD4 was reportedly close to significance. Additionally, CD4 and viral load were not related to imaging findings in the study conducted by Donald et al. (2015). Duration of HAART was not related to corpus callosum thickness (Andronikou et al. 2014) or white matter lesions (van Arnhem et al. 2013). Notably, Hoare et al. (2015) found that nutrition may play a significant role in the white matter functioning of children with HIV. Hemoglobin, total protein, and albumin levels were collected and analyzed as measures of nutritional status. They were associated with low FA and high MD values as well as low AD and high RD values, which may suggest problems with white matter integrity.

Most of the reviewed studies examined white matter, but some (Andronikou et al. 2014; Donald et al. 2015; Izbudak et al. 2013; Mariam and Assefa 2012; Nagarajan et al. 2012; Sarma et al. 2014; van Arnhem et al. 2013) also examined other neurological findings. The neurological data examined by these studies varied widely, such that limited conclusions could be drawn. Two consistent findings were basal ganglia calcification and cortical atrophy (Donald et al. 2015; Izbudak et al. 2013; Mariam and Assefa 2012).

\section{Cognitive Assessment Results}

Children with HIV of various ages were found to have difficulties with cognitive functioning compared to controls on various assessments (Hoare et al. 2012; Nagarajan et al. 2012). Hoare et al. (2015) examined children's general intelligence, hand-eye coordination and motor speed, processing speed, attention and concentration, working memory, cognitive flexibility, visuospatial processing and visual memory, semantic fluency, and inhibition. Children with HIV performed significantly more poorly than children without HIV on tests of general intelligence, visuospatial processing, visual memory, and semantic fluency. It was also reported that trends toward lower performance were observed in relation to motor functioning, processing speed, executive functioning, and cognitive flexibility. Only the correlations between FA values in the corpus callosum and superior longitudinal fasciculus and cognitive flexibility and semantic fluency were significant. Nagarajan et al. (2012) assessed three cognitive areas, including attention and processing speed, psychomotor functioning, and executive functioning and problem-solving skills. Children with HIV performed more poorly than children without HIV on tasks of attention and processing speed. None of the metabolite ratios correlated with cognitive test scores in children with HIV; however, a correlation was observed between $\mathrm{NAA} / \mathrm{tCr}$ and attention and processing speed scores for children without HIV. 
Developmental/neurocognitive issues were also noted in studies that did not compare children with HIV to children in a control group. No statistically significant difference was observed in general, locomotor, or language developmental scores between children with HIV with white matter signal abnormalities and those with HIV without signal abnormalities (Ackermann et al. 2014). The number of white matter lesions also did not correlate with developmental scores (Ackermann et al. 2014). Additionally, Andronikou et al. (2014) found that the thickness of the area of the corpus callosum associated with motor functioning was significantly correlated with general developmental scores, but no other relations among general, locomotor, or language scores and thicknesses of sections of the corpus callosum were observed.

\section{Discussion}

One aim of the present review was to better understand the recent literature on imaging in pediatric HIV because of advancements in medications and changes to medication guidelines and recommendations. Only studies published during or after 2012 were eligible for inclusion in this review in order to extend the findings of a previous review (Hoare et al. 2014) that primarily reviewed studies that had been conducted prior to 2006. The findings of the present study indicate that, although several publications have been made during this brief time period, retrospective studies are being published using data collected over large time periods, including time periods before the updates to medications and ART guidelines.

\section{HIV Treatment Effects}

It is possible that the presence of ART during infancy and early childhood, which is part of the recent changes to HIV treatment guidelines, would influence the effect of the virus on the child's rapidly developing central nervous system as well as the child's immune response to the virus. Additionally, the newer medication guidelines would lengthen the exposure to potentially neurotoxic medications. Altogether, these factors may result in neurological effects that are different from those of children who were exposed to different medications and who were treated for less time in accordance with earlier ART guidelines.

A related issue is that many studies did not clearly indicate when their data were collected. Compounding that problem is the finding that many studies also did not provide much information about the ART that the children in their samples were receiving. This lack of information makes it difficult to ascertain whether the findings of the studies are, in fact, relevant for today's population of children with HIV. More prospective studies may be needed that give necessary attention to the ART being prescribed to the children. In addition to needing more current prospective studies with adequate descriptions of ART, the research could also benefit from collecting and describing data related to medication adherence. Medication adherence can be assessed in multiple ways, and the most appropriate method for assessing adherence in a study may be dependent on a variety of factors (see Chesney 2006). Although Hoare et al. (2014) mentioned that guidelines changed in 2008 , they did not thoroughly address this issue in their review, likely because the majority of studies reviewed were published prior to the 2008 updates to WHO guidelines.

Another finding of the present review is that there are few recent studies that have examined neurological differences between children with HIV and children without HIV. Only 4 of the 11 studies included control groups. Future research could benefit from additional studies including control groups of non-infected children, including HIV-exposed children and non-exposed/non-infected children. Comparing the neurological findings of children with HIV to children without HIV but from similar backgrounds may help scholars better disentangle the neurological outcomes of HIV infection from those related to less than optimal socioeconomic, health, and educational experiences. Hoare and colleagues also noted that most studies they reviewed did not include control groups, so this appears to be a persistent finding in this body of literature.

\section{Neuroimaging and Neurocognitive Findings}

White matter appears to be a greater focus of the current body of literature on imaging in pediatric HIV than it was in the studies reviewed by Hoare et al. (2014). The findings of the present review indicate that various white matter problems have been observed in children of nearly all age ranges, many of whom were being prescribed HAART. The white matter finding in the present group of studies generally concurs with that of previous literature, as the review of Hoare et al. (2014) also indicated that white matter issues were one of the most commonly reported findings of research published prior to 2012. However, it is difficult to ascertain whether there are differences in white matter findings between the current and previous bodies of literature because of the limited number of studies examining it prior to 2012 and because of the lack of specificity in the summary of findings by Hoare et al. (2014).

Similar to the findings reported by Hoare et al. (2014), findings are still mixed about cognitive problems as well as the relation of cognitive functioning to neurological disturbances observed in pediatric populations with HIV. One study in the present review found that overall cognitive development as well as visuospatial processing, visual memory, and semantic fluency were lower in children with HIV than in children without HIV (Hoare et al. 2012). This study did not find significant difficulties with motor functioning, processing speed, executive functioning, and cognitive flexibility despite the fact that these are some of the more commonly found cognitive 
difficulties associated with HIV (Wood et al. 2009). Yet, another study included in this review (Nagarajan et al. 2012) found significantly weaker scores in attention/processing speed in children with HIV and children without HIV. The lack of consensus in the small body of literature suggests that it would be beneficial for future studies to routinely include neuropsychological assessment as part of neuroimaging studies to help identify brain-behavior relations associated with HIV.

Incorporating comprehensive neuropsychological assessment batteries into neuroimaging studies is a good practice and seems to be becoming more common. However, the results of the present review indicated that studies using cognitive assessment tended to assess only certain skills or domains of cognitive functioning, such as executive functioning and motor skills. Sherr et al. (2014) suggested in their updated review that studies examining the cognitive and developmental sequelae of HIV have become more comprehensive over time, including more measures and a greater coverage of domains. However, this does not appear to be the case for recent combination neuroimagingneuropsychological studies attempting to establish brainbehavior relations. It is therefore recommended that future neuroimaging-neuropsychological studies should include neuropsychological assessment in a way that best answers the research question. That is, studies examining wholebrain issues such as global atrophy may be best served by providing participants with a comprehensive neuropsychological battery assessing each neuropsychological domain. In contrast, studies examining a specific brain structure may find it beneficial to more deeply and thoroughly assess the range of functions related to that structure. Finally, serial neuroimaging and neuropsychological assessments may be helpful for understanding how the neurological progression of the disease, or lack thereof, affects functioning over time and developmental periods.

\section{Ecological Approach}

Future research is needed to better understand the neurological consequences of HIV as well as the links between the neurological sequelae and the behavioral functioning of children with HIV, including the influence of treatment. Importantly, children living with HIV in the present day may have access to more effective medication earlier in life, as a result of changes to ART guidelines and advancements in pharmacology. These experiences may make these children different from children with HIV from several years ago. There is a need for prospective studies involving children with HIV as well as typically developing controls or children exposed to HIV. Perhaps the most important finding of this review, however, may be the need for an ecological approach to be used in future imaging research in this area.
Ecological systems theory of Bronfenbrenner (1979) describes that there are four levels of environments that are involved in the development of a person: the microsystem, mesosystem, exosystem, and macrosystem. The microsystem is the immediate environment around the person, including the home, neighborhood, school, religious, and other settings, and the individuals within those places. The mesosystem includes the interactions between microsystem settings. For example, children who experience trauma in the home may be oppositional in the classroom. The exosystem refers to settings that the person is not actively involved in, but that influences the development of the person. Examples of exosystem settings include community resources and mass media. Finally, the macrosystem is the cultural context in which the person develops and includes the belief systems, norms, and policies that affect the individual.

Researchers have found that a range of family-, resource-, and health-related variables may be influencing the neurocognitive development of children. This explains why several other reviews (Abubakar et al. 2008; Hoare et al. 2014; Sherr et al. 2014) have made recommendations to take such variables into consideration in future research on children with HIV. We suggest, however, that it is not enough to assess some of these variables at random, as has been done in the previous body of imaging literature. Lack of a consistent research protocol is likely contributing to the mixed results obtained in previous research and the difficulty with interpreting the etiology of the neurological issues observed in children with HIV (Hoare et al. 2014). It may also make it difficult to compare studies completed in industrialized countries to those completed in developing countries and vice versa. We recommend using an ecological approach to conceptualize the challenges facing children and families with HIV and to use this framework for selecting variables for inclusion in research related to children with HIV. Doing so could provide a consistent framework for future research that takes into account the broad interactions of variables contributing to developmental outcomes for children with HIV. It also would allow for sufficient information to be collected and disseminated so that accurate population-based comparisons can be made between studies conducted in industrialized and developed countries.

An example of the ecological approach applied to children with HIV follows. Children with HIV experience many individual factors related to HIV that need to be taken into account. These may include viral load, immune system status, and clinical stage, among others. Individual factors more distally or unrelated to HIV that influence neurocognitive development that may need to be assessed may include exposure to substances and secondary infections in utero, prematurity and birth weight, and early experiences of trauma because these are all variables known to influence brain development (Birk Irner 2012; Hack et al. 2000; Teicher et al. 2003). It is not 
sufficient to assess just these variables, however, because these children exist in multiple microsystems that also influence their development. Microsystem factors may include educational experiences and caregiving issues (e.g., orphanhood, quality of caregiving). Research indicates that these are important issues for children affected by HIV (Foster and Williamson 2000). The interaction of variables within the domains of the microsystem, representing the mesosystem, may also need to be taken into account. Caregivers of children with HIV may experience difficulties with maintaining employment and accessing adequate health care and nutrition (Williams et al. 2011). These represent exosystem factors that would likely affect the development of a child with HIV, which highlight the need for the inclusion of variables related to socioeconomic status of the family and nutritional and health status of the child. Finally, countries and international organizations create policies related specifically to the management of HIV (e.g., guidelines for ART). Such macrosystem issues would be related to the development of children with HIV and therefore highlight the need to assess children's ART regimens, ART duration, and ART adherence. Another important macrosystem issue affecting populations with HIV may be the stigma toward HIV embedded within a culture, which affects mental health status and medication adherence (Byakika-Tusiime et al. 2009) and, therefore, may need to be assessed in relation to its effect on children's development. In sum, an ecological approach may be useful for thinking about factors affecting the neurocognitive development of children with HIV. If used to conceptualize the neurocognitive development of children with HIV, it could result in consistent and comprehensive selection of variables to be assessed in future imaging studies.

Because the studies published to date have not fully reported on important ecological data, it is difficult to fully understand the nature of the neurological implications of HIV. If future studies were able to do so, we may better capture the manipulable variables important to optimizing treatment and outcomes for this vulnerable population. Adjustments could be applied not only within children medically (e.g., HAART treatment, nutrition) but also within families, communities, and cultures, which bear an influence on the neural development of children. We suggest that by adopting an ecological model, our ability to recognize symptoms, predict outcomes, and treat would be strengthened.

\section{Conclusion}

A small number of articles were available for review in the present study. It remains difficult to determine the etiology of neurological issues associated with pediatric HIV because of the inconsistent and limited inclusion of important descriptive variables in imaging studies. The findings suggest that children with HIV at nearly all age ranges and on HAART for prolonged periods of time may have white matter volume loss (Donald et al. 2015; Sarma et al. 2014), poor white matter integrity (Hoare et al. 2012), and white matter lesions (Ackermann et al. 2014; Donald et al. 2015; Izbudak et al. 2013; van Arnhem et al. 2013). Other neurological problems such as basal ganglia calcification and cortical atrophy appear to be consistent in the research on pediatric HIV (Donald et al. 2015; Hoare et al. 2014; Izbudak et al. 2013; Mariam and Assefa 2012). Future research that addresses the issues of the existing studies is needed to better understand the challenges experienced by children with HIV, which will hopefully ultimately lead to better interventions. It is recommended that an ecological approach be used to conceptualize the neurocognitive development of children with HIV in future imaging studies.

\section{Compliance with Ethical Standards}

Ethics Statement No animal or human studies were carried out by the authors for this article.

Funding No funding was secured for this study.

Conflict of Interest The authors declare that they have no competing interests.

\section{References}

Abubakar, A., Van Baar, A., Van de Vijver, F. J. R., Holding, P., \& Newton, C. R. J. C. (2008). Paediatric HIV and neurodevelopment in sub-Saharan Africa: a systematic review. Tropical Medicine and International Health, 13, 880-887. doi:10. 1111/j.1365.2008.02079.x.

Ackermann, C., Andronikou, S., Laughton, B., Kidd, M., Dobbels, E., Innes, S., \& Cotton, M. (2014). White matter signal abnormalities in children with suspected HIV-related neurologic disease on early combination antiretroviral therapy. The Pediatric Infectious Disease Journal, 33, e207-212. doi:10.1097/INF. 0000000000000288.

Andronikou, S., Ackermann, C., Laughton, B., Cotton, M., Tomazos, N., Spottiswoode, B., \& Pettifor, J. M. (2014). Correlating brain volume and callosal thickness with clinical and laboratory indicators of disease severity in children with HIV-related brain disease. Child's Nervous System, 30(9), 1549-1557. doi:10.1007/s00381-0142434-3.

Andronikou, S., Ackermann, C., Laughton, B., Cotton, M., Tomazos, N., Spottiswoode, B., \& Pettifor, J. M. (2015). Corpus callosum thickness on mid-sagittal MRI as a marker of brain volume: a pilot study in children with HIV-related brain disease and controls. Pediatric Radiology, 45(7), 1016-1025. doi:10.1007/s00247-014-3255-y.

Birk Irner, T. (2012). Substance exposure in utero and developmental consequences in adolescence: a systematic review. Child Neuropsychology, 18(6), 521-549. doi:10.1080/09297049.2011. 628309.

Bronfenbrenner, U. (1979). The ecology of human development: experiments by nature and design. Cambridge: Harvard University Press. 
Byakika-Tusiime, J., Crane, J., Oyugi, J. H., Ragland, K., Kawuma, A., Musoke, P., \& Bangsberg, D. R. (2009). Longitudinal antiretroviral adherence in HIV+ Ugandan parents and their children initiating HAART in the MTCT-Plus family treatment model: role of depression in declining adherence over time. AIDS and Behavior, 13, S82S91. doi:10.1007/s10461-009-9546-x.

Chesney, M. A. (2006). The elusive gold standard: future perspectives for HIV adherence assessment and intervention. Journal of Acquired Immune Deficiency Syndromes, 43, S149-S155. doi:10.1097/01. qai.0000243112.91293.26.

Donald, K. A., Walker, K. G., Kilborn, T., Carrara, H., Langerak, N. G., Eley, B., \& Wilmshurst, J. M. (2015). HIV encephalopathy: pediatric case series description and insights from the clinic coalface. AIDS Research and Therapy, 12, 1-10. doi:10.1186/s12981-014-0042-7.

Ellis, R. J., Calero, P., \& Stockin, M. D. (2009). HIV infection and the central nervous system. Neuropsychological Review, 19, 144-151. doi:10.1007/s11065-009-9094-1.

Foster, G., \& Williamson, J. (2000). A review of current literature of the impact of HIV/AIDS on children in sub-Saharan Africa. AIDS, 14, S275-S284. Retrieved from http://www. hs r c.ac.za/uploads/page Content/1670/ AreivewofcurrentliteratureontheimpactoforphansinAfrica.pdf.

Gogtay, N., Giedd, J. N., Lusk, L., Hayashi, K. M., Greenstein, D., Vaituzis, A. C., \& Thompson, P. M. (2004). Dynamic mapping of human cortical development during childhood through early adulthood. Proceedings from the National Academy of Sciences, 101(21), 8174-8179. doi:10.1073/pnas.0402680101.

Hack, M., Wilson-Costello, D., Friedman, H., Taylor, G., Schluchter, M., \& Fanaroff, A. A. (2000). Neurodevelopment and predictors of outcomes of children with birth weights of less than $1000 \mathrm{~g}$. Archives of Pediatrics and Adolescent Medicine, 154(7), 725-731. doi:10.1001/ archpedi.154.7.725.

Hoare, J., Fouche, J.-P., Phillips, N., Joska, J. A., Donald, K. A., Thomas, K., \& Stein, D. J. (2015). Clinical associations of white matter damage in cART-treated HIV-positive children in South Africa. Journal of Neurovirology, 21, 120-128. doi:10.1007/s13365-014-0311-1.

Hoare, J., Fouche, J.-P., Spottiswoode, B., Donald, K., Philipps, N., Bezuidenhout, H., \& Stein, D. (2012). A diffusion tensor imaging and neurocognitive study of HIV-positive children who are HAART-naive "slow progressors". Journal of Neurovirology, 18, 205-212. doi:10.1007/s13365-012-0099-9.

Hoare, J., Ransford, G. L., Phillips, N., Amos, T., Donald, K., \& Stein, D. J. (2014). Systematic review of neuroimaging studies in vertically transmitted HIV positive children and adolescents. Metabolic Brain Disease, 29, 221-229. doi:10.1007/s11011-013-9456-5.

Izbudak, I., Chalian, M., Hutton, N., Baskaran, V., Jordan, L., Siberry, G. K., \& Agwu, A. L. (2013). Perinatally HIV-infected youth presenting with acute stroke: progression/evolution of ischemic disease on neuroimaging. Journal of Neuroradiology, 40, 172-180. doi:10. 1016/j.neurad.2012.08.001.

Liner, K. J., II, Ro, M. J., \& Robertson, K. R. (2010). HIV, antiretroviral therapies, and the brain. Current HIV/AIDS Reports, 7, 85-91. doi: 10.1007/s11904-010-0042-8.

Mariam, A. G., \& Assefa, G. (2012). Clinical and neuroimaging profile of HIV-1 encephalopathy in infancy and childhood in a sub-Saharan African country. Ethiopian Medical Journal, 50(3), 337-347.

Nagarajan, R., Sarma, M. K., Thomas, M. A., Chang, L., Natha, U., Wright, M., \& Keller, M. A. (2012). Neuropsychological function and cerebral metabolites in HIV-infected youth. Journal of Neuroimmune Pharmacology, 7, 981-990. doi:10.1007/s11481012-9407-7.

Patel, K., Hernan, M. A., Williams, P. L., Seeger, J. E., McIntosh, K., van Dyke, R. B., \& Seage, G. R., III. (2008). Long-term effectiveness of highly active antiretroviral therapy on the survival of children and adolescents with HIV infection: a 10-year follow-up study. Clinical Infectious Diseases, 46, 507-515. doi:10.1086/526524.

Robertson, K., Liner, J., \& Meeker, R. B. (2012). Antiretroviral neurotoxicity. Journal of Neurovirology, 18, 388-399. doi:10.1007/ s13365-012-0120-3.

Sarma, M. K., Nagarajan, R., Keller, M. A., Kumar, R., Nielsen-Saines, K., Michalik, D. E., \& Thomas, M. A. (2014). Regional brain gray and white matter changes in perinatally HIV-infected adolescents. NeuroImage: Clinical, 4, 29-34. doi:10.1016/j.nicl.2013.10.012.

Sherr, L., Croome, N., Parra Castaneda, K., Bradshaw, K., \& Herrero Romero, R. (2014). Developmental challenges in HIV infected children - an updated systematic review. Children and Youth Services Review, 45, 74-89. doi:10.1016/j.childyouth.2014.03.040.

Sowell, E. R., Thompson, P. M., Tessner, K. D., \& Toga, A. W. (2001). Mapping continued brain growth and gray matter density reduction in dorsal frontal cortex: inverse relationships during post adolescent brain maturation. The Journal of Neuroscience, 21(22), 8819-8829. Retrieved from: http://www.jneurosci.org/content/21/22/8819.full. pdf + html.

Teicher, M. H., Andersen, S. L., Polcari, A., Anderson, C. M., Navalta, C. P., \& Kim, D. M. (2003). The neurobiological consequences of early stress and childhood maltreatment. Neuroscience and Biobehavioral Reviews, 27, 33-44. doi:10.1016/ S0149-7634(03)00007-1.

van Arnhem, L. A., Bunders, M. J., Scherpbier, H. J., Majoie, C. B. L. M., Reneman, L., Frinking, O., \& Pajkrt, D. (2013). Neurologic abnormalities in HIV-1 infected children in the era of combination antiretroviral therapy. PLoS One, 8(5), e64398. doi:10.1371/journal.pone. 0064398.

Whitehead, N., Potterton, J., \& Coovadia, A. (2013). The neurodevelopment of HIV-infected infants on HAART compared to HIV-exposed but uninfected infants. AIDS Care: Psychological and Sociomedical Aspects of AIDS/HIV, 26(4), 497-504. doi:10. 1080/09540121.2013.841828.

Williams, B., Amico, K. R., \& Konkle-Parker, D. (2011). Qualitative assessment of barriers and facilitators to HIV treatment. Journal of the Association of Nurses in AIDS Care, 22, 307-312. doi:10.1016/j. jana.2010.11.001.

Wood, S. P., Moore, D. J., Weber, E., \& Grant, I. (2009). Cognitive neuropsychology of HIV-associated neurocognitive disorders. Neuropsychology Review, 19, 152-168. doi:10.1007/a11065-0099102-5.

World Health Organization. (2006). Antiretroviral therapy of HIV infection in infants and children: Towards universal access. Retrieved from http://www.who.int/hiv/pub/paediatric/infants/en/

World Health Organization. (2007). WHO case definitions of HIV for surveillance and revised clinical staging and immunological classification of HIV-related disease in adults and children. Geneva: WHO Press, World Health Organization.

World Health Organization. (2008). Report of the WHO Technical Reference Group. Paediatric HIV/ART Care Guideline Group meeting. Retrieved from http://www.who.int/hiv/pub/paediatric/WHO Paediatric ART guideline rev mreport 2008.pdf

World Health Organization. (2010). Antiretroviral therapy for HIV infection in infants and children: towards universal access. Retrieved from http://www.who.int/hiv/pub/paediatric/infants2010/en/

World Health Organization. (2013). Consolidated guidelines on the use of antiretroviral drugs for treating and preventing HIV infection. Retrieved from http://www.who.int/hiv/pub/guidelines/arv2013/ download/en/

World Health Organization. (2015a). Antiretroviral therapy. Retrieved from http://www.who.int/topics/antiretroviral_therapy/en/

World Health Organization. (2015b). Treatment of children living with HIV. Retrieved from http://www.who.int/hiv/topics/paediatric/hivpaediatric-infopage/en/ 